\title{
P01-1-6 Poster session
}

\section{Involvement of ROS/8-nitro-cGMP signals in cerebellar synaptic plasticity in mice}

\section{Sho Kakizawa ${ }^{1}$, Shogo Endo ${ }^{2}$}

\author{
${ }^{I}$ Department of Biological Chemistry, Kyoto University, Graduate School of Pharmaceutical Sciences, Japan, ${ }^{2}$ Memory \\ Neuroscience Section, Aging Neuroscience Research Team, Tokyo Metropolitan Institute of Gerontology, Japan
}

Reactive oxygen species (ROS), such as super oxide and hydrogen peroxide, have long been considered as harmful molecules within biological systems, since accumulation of oxidative modification are often observed in pathological states. However, recent observations demonstrating expression of ROS synthase, including NADPH oxidase (Nox) and dual oxidase (Duox), in various tissues suggest possible involvement of ROS in physiological events. Expressions of Nox and Duox are also reported in the central nervous systems (CNS). However, physiological role(s) of ROS in brain has yet to be determined.

Synaptic plasticity in the CNS is widely accepted as the cellular basis of memory and learning. Long-term depression at parallel fiber-Purkinje cell synapse (PF-LTD) is considered to be the cellular substrate of cerebellar-dependent motor learning such as eyeblink conditioning and opto-kinetic reflex. Various molecules have been indicated to be involved in the induction and expression of PF-LTD so far. However, molecular mechanism of the long-term memory has not been fully understood.

8-nitro-cGMP, produced from GTP, nitric oxide (NO) and ROS, has unique properties. Because the molecule is resistant to phosphodiesterase which catalyze cGMP, 8-nitro-cGMP has longer life and potential to activate protein kinase $\mathrm{G}(\mathrm{PKG})$ for longer time than cGMP. In addition, 8-nitro-cGMP has also potential to activate PKG through a chemical modification, guanylation. Because this chemical modification is pretty stable, the fact also suggests longer activation of PKG by 8-nitro-cGMP than cGMP. Because PKG is indicated to be essential for the induction of PF-LTD, these matters suggest that 8 -nitro-cGMP is one of the candidates of long-term molecular memory.

Therefore, in the present study, we examined functional roles of ROS/8-nitro-cGMP signals in PF-LTD induction. Acute cerebellar slices were prepared from young-adult mice, and excitatory postsynaptic currents of PF synapses (PFEPSC) were recorded from Purkinje cells. PF-LTD was impaired by application of 8-nitro-cGMP analog, 8-nitro-cGMPS. PF-LTD was also inhibited by ROS removal (by superoxide dismutase and catalase) or Nox inhibitor, apocynin. These results suggest essential roles of ROS/8-nitro-cGMP signals in the induction of PF-LTD in mouse cerebellum. 